\title{
Expertengespräch: Forschung und Entwicklung in der Phytotherapie - Teil 2
}

Die grössten Fortschritte hat die Phytotherapie im 19. Jahrhundert gemacht. Von der Begeisterung, mit der sich Apotheker und Chemiker in die Analyse der Naturwirkstoffe stürzten, lässt sich Dr. Michael Kessler, Leiter des Pharmaziehistorischen Museums in Basel, heute noch anstecken.

Wo wäre die moderne Medizin ohne Phytotherapeutika?

Kessler: Ohne Phytotherapeutika gäbe es sie wahrscheinlich gar nicht. Die moderne Medizin hat sich erst im 19. Jahrhundert entwickelt. Wir verstehen unter moderner Medizin die Medizin, die auf dem zellularpathologischen Modell von Virchow aufgebaut ist, und nicht mehr die klassische Medizin, die in der Humoralpathologie begründet ist, der alten Säftelehre. Dieser Übergang findet im 19. Jahrhundert statt. Krankheit ist nicht länger ein Missverhältnis der Säfte, wie man das vorher dachte, sondern eine Fehlfunktion der Zellen. Das ist der Übergang von der Humoralpathologie in die Zellularpathologie, die wir eigentlich als moderne Medizin bezeichnen. In der gleichen Zeit, in der ersten Hälfte des 19. Jahrhunderts, findet auch der Übergang von einer empirisch zufälligen Therapie mit Pflanzen zu einer rationaleren Phytotherapie statt. In der zweiten Hälfte des 19. Jahrhunderts folgt dann der Übergang zur Entwicklung pharmakologischer Präparate, die dann auch aus der synthetischen Chemie kommen.

Die Phytotherapie und die Pharmakologie entwickeln sich schliesslich durch methodische Fortschritte in der synthetischen Chemie auseinander?

Ja, sicher. Der Anfang der Phytotherapie im modernen Sinn ist die aufkommende Alkaloidchemie im 19. Jahrhundert. 1806 ist die Schnittstelle, weil man damals zum ersten Mal einen pflanzlichen Wirkstoff rein dargestellt hat. Dem Apotheker Friedrich Wilhelm Sertürner gelang es, Morphium aus Opium zu extrahieren. Diese Leistung ist so wichtig, weil es damit möglich wurde, solche Stoffe überhaupt zu dosieren, was man vorher nicht konnte. Man hat Opium schon lange verwendet und wusste, dass es Schmerzen stillen kann, aber man konnte es eigentlich nicht dosieren, da man nicht wusste, wie viel Morphium da drin ist, und man die wirksame Substanz auch gar nicht kannte. Der Gehalt an Morphin schwankt sehr stark im Opium; man hat einen alkoholischen Auszug gemacht, eine Tinktur, aber ohne definierten Gehalt. Möglich wird das Dosieren erst mit der Alkaloidchemie in der ersten Hälfte des 19. Jahrhunderts. Entscheidend ist, dass es damit möglich ist, Therapien zu standardisieren und eine Beziehung zwischen Dosierung und Wirkung herzustellen.

Die Möglichkeit, kausale Beziehungen herzustellen, begründet den

Siegeszug dieser Stoffe?

Genau, und nicht unterschätzen darf man, dass man plötzlich versteht, dass diese pflanzlichen Wirkstoffe quantitativ erfasst werden müssen, damit man sie dosieren kann. Nach Sertürner, der seine Ergebnisse erst 1816 publiziert hat, weil man ihn als Apotheker nicht ernst genommen hat, ging die Entwicklung der ganzen Alkaloidchemie sehr schnell. Es wurde möglich, erstens diese Stoffe in standardisierten Therapien anzuwenden und zweitens auch industriell zu produzieren. Das ist der Anfang der modernen Arzneimittelentwicklung.

Damit richtet sich die Konzentration nicht mehr auf ein Wirkstoffgemisch, sondern auf einzelne Bestandteile?

Ja, das ist ein ganz wichtiger Punkt, dass man sich von dieser polypharmazeutischen Idee entfernt. Die ging davon aus, dass man möglichst viele Wirkstoffe miteinander verwenden sollte, damit man eine möglichst gute Wirkung hat. Allerdings hatte schon Paracelsus gefordert, dass man einzelne Stoffe verwenden soll und nicht Mischungen. Man hat das natürlich lange Zeit nicht akzeptiert, weil man eben auch technisch sowie chemisch die Möglichkeiten nicht hatte. Das hat sich natürlich sehr stark mit der Materialtechnologie und ihrem Fortschritt im 18. Jahrhundert verändert. Im 19. Jahrhundert hat sich das dann sehr stark bemerkbar gemacht. Das war die Blütezeit der Phytotherapie, denn in dieser Zeit waren die verwendeten Wirkstoffe pflanzlich und die Mittel standardisierte Extrakte.

Was konnte man damals eigentlich sehr gut mit Phytotherapeutika behandeln?

Zum Beispiel Schmerzen mit Morphium; dass man Malaria mit Chinin behandeln kann, dass man Atropin einsetzen kann, wusste man. Das waren grosse Fortschritte, die die 
Grundlage der pharmakologischen Seite der modernen Medizin wurden. Die «chemischen» Wirkstoffe waren im frühen 19. Jahrhundert meistens Metallsalze - z.B. Quecksilber- oder Kupfersalze. Man hat sie gerne verwendet, weil sie starke Wirkungen zeigten, aber oft überdosiert. Man hat gesagt, die Patienten der Therapien leiden mit. Und die aufkommenden Phytotherapeutika, die waren eben viel milder als diese Metallsalze, die man vorher gern verwendet hat. Man hatte ausser mit den Metallsalzen keine antibiotische Therapie bis ins 20. Jahrhundert hinein. Erst dann kam das Penicillin, und im Zweiten Weltkrieg kamen dann die Chemotherapeutika, die auch Arsen enthalten, aber eben in einer anderen Form.

Mit dem Fortschritt der Chemie begann man, die Wirkstoffe zu optimieren?

In der zweiten Hälfte des 19. Jahrhunderts fängt man an, synthetische Stoffe herzustellen, die wirken, aber eine sanfte Wirkung haben. Diese chemischen Substanzen beginnen, die Phytotherapeutika wieder etwas zurückzudrängen. Denken Sie z.B. an die Entwicklung des Aspirins - auch eine Erfindung des späten 19. Jahrhunderts. Man hat die pflanzliche Basis genommen und sie dann weiter entwickelt. Das Heroin ist so entstanden. Es war als starkes Schmerzmittel gedacht, das nicht abhängig macht.

Wie hat man damals Wirkstoffe gefunden? Es gab ja keine Screening-Verfahren, wie wir sie heute kennen. Hat man sich da des Volkswissens bedient?

Man hat schon aus der Empirie, also aus der Erfahrung, viel gewusst. Es war bekannt, dass etwas Opium schmerzlindernd wirkt. Als man dann begriffen hat, dass dort ein Stoff vorhanden sein muss, der diese Wirkung hat, hat man begonnen, danach zu suchen. Auch die Wirkung von Tollkirschenblättern, aus denen dann das Atropin extrahiert wurde, kannte man und wusste, dass Chinarinde bei Malaria hilft und fiebersenkend wirkt. Schliesslich entdeckte man das Chinin.

\section{Wie wurde das Wissen über}

die Heilpflanzen ausgetauscht und weitergegeben?

Die Klöster und die Texte aus der Klassik haben hier eine zentrale Rolle gespielt. Die Arzneikundebücher der europäischen Antike sind hier überliefert worden. Aber es gibt natürlich dann auch neuere Entdeckungen in den Klöstern, wie etwa die Pflanzenbücher von Otto Brunfels oder Hieronymus Bock, in denen neue Heilkräuter beschrieben werden.

Und diese Tradition aus der Antike ist von dem, was man so allgemein als Volksmedizin bezeichnet, bereichert worden?

Das sieht man in den Kräuterbüchern des 16./17. Jahrhunderts, in denen eben nicht mehr nur das klassische Spektrum der Heilpflanzen aufgeführt ist, sondern auch Pflanzen aus der Volksmedizin auftauchen.

Die Phytotherapie ist ein Kind des sehr rationalen 19. Jahrhunderts und wird heute oft von einer eher wissenschaftsskeptischen Seite für sich reklamiert, der die moderne Medizin nicht behagt.

Diesen Konflikt gab es schon seit dem frühen 19. Jahrhundert mit der Homöopathie, die sich ja gegen die Schulmedizin gestellt hat. Sie war damals sehr populär, weil sie eine sanfte Therapie war. Damit stand sie im Gegensatz zur Behandlung mit pflanzlichen Extrakten, die damals auch eine «bittere Medizin» war, wie man das ja auch genannt hat. Die sanfte Alternative war Homöopathie. Dann kommen in der zweiten Hälfte des 19. Jahrhunderts synthetische Derivate von pflanzlichen Wirkstoffen wie z.B. Aspirin auf den Markt, die dann eben nebenwirkungsarm sind, aber funktionieren. Sie drängen die Homöopathie wieder zurück. Im 20. Jahrhundert hat man zunächst als Chemotherapeutika synthetische

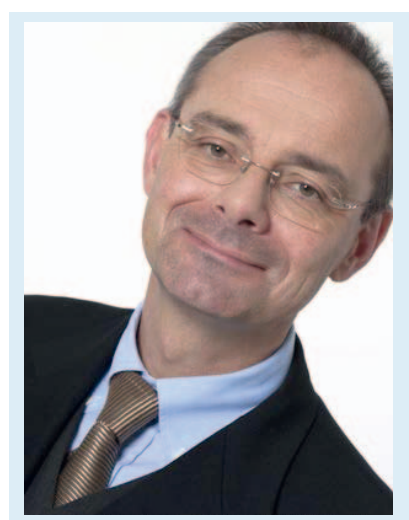

\section{Dr. Michael Kessler}

(michi.kessler@unibas.ch) ist Direktor des Pharmaziehistorischen Museums der Universität Basel (www.pharmaziemuseum.ch).

«Ohne Phytotherapeutika gäbe es die moderne Medizin wahrscheinlich gar nicht.»

Substanzen entwickelt - wie organische Arsenverbindungen und Sulfonamide. Die Antibiotika wie Penicillin - die Peptide sind - kommen dann erst in der Zeit des Zweiten Weltkriegs.

\section{Welcher phytotherapeutische} Wirkstoff aus den letzten 2000 Jahren ist für Sie der interessanteste?

Spannend finde ich die grossen, wichtigen Pflanzen, die Alkaloide enthalten. Sicher ist das Opium mit dem Morphium und all den anderen Derivaten, wie Heroin, bedeutend - und nicht nur medizinisch. Ihre Wirkung zieht sich durch die ganze Geschichte von der minoischen Kultur bis heute. Denken Sie an den Opiumkrieg in China oder beim Chinin an die längst vergessenen Konflikte: Die Hoffnung auf die Entwicklung einer Synthese des Chinins durch die Engländer, weil sie vom Wohlwollen der Niederländer wegen der Chinarindenimporte abhängig wurden. Die Krone sah das als sehr ungünstig an und hat versucht, das Chinin synthetisch herstellen zu lassen. Prinz Albert, der Mann Königin Victorias, hat einen deutschen Gelehrten nach England geholt, der den Auftrag hatte, Chinin künstlich herzustellen. Das gelingt nicht, aber er findet eine Methode, künstliche Farben zu synthetisieren: die Anilinfarben.

Interview: Oliver Klaffke 